\title{
Visceral Larva Migrans Syndromes Associated with Toxocariasis: Epidemiology, Clinical and Laboratory Aspects of Human Toxocariasis
}

\author{
Elaine Alvarenga de Almeida Carvalho • \\ Regina Lunardi Rocha
}

Published online: 8 January 2014

(C) Springer International Publishing AG 2014

\begin{abstract}
Toxocariasis is caused by the helminth Toxocara spp. Its prevalence is underestimated in many settings and affects primarily children due to their vulnerability. Toxocariasis may be an asymptomatic infection or it may progress to cause severe clinical forms. The aim of this paper is to describe the epidemiology, risk factors, clinical and laboratory diagnosis of visceral larva migrans that manifest in the liver, lung, or central nervous system. It is recommended that antiparasitic treatment should be instituted even in asymptomatic patients to prevent migration of the larva to vital organs. There is a lack of awareness of the population of this parasitic infection and also among clinicians regarding the clinical manifestations of toxocariasis. The lack of public health preventive measures leads to its high prevalence in many settings, usually impoverished areas in tropical settings. Thus, it is imperative to prevent transmission and to provide early treatment of asymptomatic or symptomatic cases to prevent complications and late sequelae of this parasitic infection.
\end{abstract}

Keywords Toxocara canis · Epidemiology · Child health . Serology · ELISA · Antigens TES · Hypoechoic lesions · Liver - Uveitis · Eosinophilia · Immunoglobulin E . Ultrasound fundoscopy $\cdot$ Onychophagia $\cdot$ Dogs $\cdot$ Prevalence . Albendazole $\cdot$ Total immunglobulin $\mathrm{G}$

\section{Introduction}

Toxocariasis is considered a widely prevalent infection with a worldwide distribution, frequently underreported and thus

E. A. de Almeida Carvalho $(\varangle) \cdot$ R. L. Rocha

Department of Pediatrics, School of Medicine UFMG, 190 Prof.

Alfredo Balena Avenue, Suite 267, Santa Efigênia, Belo Horizonte,

Minas Gerais, Brazil 31130-100

e-mail: elaineacarvalho@terra.com.br classified as a neglected parasitic infection $[1 \bullet \bullet$. The term visceral larva migrans (VLM) was given to this condition due to the aberrant migration of the larvae in human tissues with incomplete maturation producing clinical manifestations in the eye, lung, and liver [2••]. Human infection occurs through the accidental ingestion of embryonated eggs of Toxocara canis excreted by dogs and less frequently caused by Toxocara cati from its excretion by cats $[2 \bullet \bullet]$. Children are more likely to become infected and develop clinical manifestations due to the immaturity of their immune system [3]. In addition, children are also at a higher risk of exposure to Toxocara spp. while playing at sites contaminated with animal excreta $[4,5 \cdot]$ such as tanks of sand in parks, daycare centers and schools, lawns and playgrounds $[4,6,7 \cdot]$. Personal habits such as onychophagia $[8 \cdot \bullet, 9 \cdot \bullet]$ and geophagy (more frequent in infants and pre-school children) or during close contact with infected dogs are also considered important mechanisms of acquisition of infection [4]. Furthermore, low socioeconomic status, which in many settings is associated with poor sanitation and hygiene, and having infected domestic $\operatorname{dogs}\left[8 \bullet^{\bullet}\right]$ are considered important risk factors for the development of the disease. Clinical manifestations may vary from asymptomatic to severe clinical presentations. The lack of controlled studies using similar diagnostic methods may be underestimating the prevalence of this infection of universal distribution. For this reason, there is a need to improve our understanding of the epidemiology, clinical presentation, laboratory diagnosis and the importance of treatment and prevention of this clinical syndrome.

\section{Epidemiology}

Toxocara eggs are not infectious and do not embryonate when excreted in the feces of dogs and cats. Dogs may become infected congenitally or perinatally [10]. Thus, man may 
become an accidental host by ingestion of embryonated eggs of Toxocara from contaminated soil, vegetables and unwashed hands, undercooked muscles or viscera of paratenic hosts such as chicken, sheep and cattle. They hatch in the intestine and penetrate through the small intestine; the liver may become infected as they pass through the portal system and hepatic sinusoids, and from there disseminate to other organs. In this manner, VLM may manifest by one or many clinical syndromes that include cardiac, pulmonary, neurological, ocular and hepatic.

Depending on the type of soil and environmental conditions, such as temperature and humidity, the eggs develop to the infective stage and can survive for at least 1 year under optimal conditions. Some authors found no association with temperature and number of eggs; however, contamination was more frequent during the fall season [11]. Toxocara infection in dogs has been detected by microbiological methods in several studies in different settings with a prevalence ranging from 10 to $100 \%$ when evaluating environmental soil samples around public parks and playgrounds $[4,7 \bullet, 11-13,14 \cdot, 15]$. Surveillance studies in elementary schools have demonstrated a prevalence that ranges from 9.2 to $33.3 \%[4,7 \cdot, 13,16 \bullet \cdot]$. Greater contamination of sand and lawn has been identified in periurban areas and slums [15].

There was no statistical difference in contamination by Toxocara spp. of sand and grass between schools and public squares $[7 \cdot, 15]$. It seems that sand suffers more influence from climate and that can contribute to contamination [13].

The incidence of toxocariasis remains unknown and the prevalence in children is considered to be underestimated [5 ${ }^{\circ}$. Prevalence of toxocariasis ranges from 4.2 to $51.6 \%$ in children in different studies and in different settings $[1 \bullet \bullet, 4$, $7 \cdot, 8 \bullet \bullet, 9 \bullet \bullet, 12,17,18 \bullet \bullet, 19 \bullet, 20]$, and of $7-46.3 \%$ in adults $[21 \cdot, 22,23]$. Experimental studies in mice have shown that immunosuppression establishes a significant larval load, which may explain the higher susceptibility of younger children with immature immune systems to suffer a higher burden of disease [3]. Prevalence studies were between 0 and $16 \%$, with the highest prevalence among schoolchildren $[4,7 \bullet, 8 \bullet$, $9 \bullet \bullet, 17,18 \bullet \bullet, 19 \bullet, 20,24 \bullet]$ older than 10 years $[1 \bullet \bullet$ and male $[7 \bullet, 8 \bullet \bullet, 9 \bullet \bullet, 18 \bullet \bullet, 19 \bullet, 20,22,24 \bullet]$, as also reported by E.A.A. Carvalho and R.L. Rocha (in press) in a cross-sectional study of 37 children with the use of ELISA IgG anti-T. canis $>640$. Other risk factors for infection included: presence of dogs in the household $[8 \bullet \bullet, 9 \bullet \bullet, 11]$, dogs younger than 1 year $[1 \bullet \bullet$, cats $[9 \bullet \bullet]$, dogs sleeping in close contact with the owner [1 $\bullet \bullet$, low household income $[8 \bullet \bullet, 9 \bullet \bullet, 21 \bullet]$ unfiltered water in the household, peri-urban slums [8••], and a history of contact with contaminated soil [21•]. The higher frequency of contact with contaminated soil on squares, regardless of season, lawn and sand around the homes (even with a lower rate of contamination) showed significant association with toxocariasis [4]. But Núñez et al. [1••] found no association between playgrounds, contact with dogs and puppies $<1$ year, frequency of contact with dogs, not washing hands before meals, consumption of fruits and vegetables unwashed, and consumption of raw meat. Santarém et al. [9・•] found no statistical difference in the prevalence among middle-class children and underprivileged. Protective factors were higher maternal education and being female. Other descriptive studies have shown that most children with toxocariasis maintain contact with dogs $[1 \bullet \bullet, 7 \bullet]$ and puppies ( $<6$ months) $[1 \bullet \bullet]$, do not wash their hands before meals $[1 \bullet \bullet$, have a history of playing in parks or playgrounds $[9 \cdot \bullet]$ and there is low schooling among their mothers $[8 \cdot \bullet, 20]$. A study by E.A.A. Carvalho and R.L. Rocha (unpublished) conducted in Belo Horizonte, Brazil identified that among children with documented toxocariasis, $71.4 \%$ of households had three or more children, $70.6 \%$ of households had family income less than or equal to the minimum wage, $94.3 \%$ of children had contact with sand or earth on a frequent basis, and $97 \%$ had domestic dogs; $91.4 \%$ had puppies ( $<6$ months), and of these, only $23.5 \%$ of domestic dogs had received effective anti-parasitic prophylaxis.

\section{Clinical Manifestations}

Toxocariasis is often subclinical or self-limiting. However, in many cases it may lead to a wide range of clinical presentations that depend on the degree of involvement of host tissue by the larvae [9••]. VLM is a systemic disorder that may compromise the lungs, heart and liver. It is compartmentalized (ocular larva migrans (OLM) or neurological (LMN); there is a covert form of toxocariasis, and asymptomatic toxocariasis [2••].

VLM is more frequent in children under three years and may present with significant eosinophilia $[1 \bullet \bullet, 9 \bullet \cdot, 16 \bullet \bullet, 22]$ with levels reaching 20,000 eosinophils $/ \mathrm{mm}^{3}$ (E.A.A. Carvalho, in press) and leukocytosis $>10,000$ cells $/ \mathrm{mm}^{3}$. Clinical signs include [2*0] hepatomegaly in $18 \%$, of cases [24•], peripheral lymphadenopathy in $37 \%$ [24•], and elevation of total $\operatorname{IgE}[16 \bullet \bullet, 22,24 \bullet]$, and $\operatorname{IgG}$ total in more than $50 \%$ of cases $[16 \bullet, 22,24 \cdot]$. In addition to laboratory abnormalities, hepatic granulomas may be identified in abdominal ultrasound and presenting as hypoechoic lesions and periportal lymphadenopathy. There are also descriptions of toxocariasis liver abscess in children [2••].

A cross-sectional study of 37 children with serological confirmation of diagnosis (ELISA >640) conducted by E.A.A. Carvalho and R.L. Rocha (in press) found a higher prevalence of infection in males $(57 \%)$. In this study, clinical manifestations included abdominal pain in $30 \%$ of cases, hepatomegaly in $22 \%$, asthma in $43 \%$, peripheral lymphadenopathy in $19 \%$ in peripheral lymphadenopathy, splenomegaly in $27 \%$, ocular findings in $3 \%$, hypoechoic liver lesions by ultrasound and/or mesenteric lymphadenopathy or 
periportal or peripancreatic lymphadenopathy in $30 \%$ (Fig. 1). Liver abscess occurred in $5.4 \%$ of cases. Salient laboratory abnormalities included the presence of leukocytosis in $16 \%$ of cases, eosinophilia in $89 \%$ with hypereosinophilia $\left(>1,000\right.$ cells $\left./ \mathrm{mm}^{3}\right)$ in $68 \%$; high total IgE for age in $97 \%$ and hyper $\operatorname{IgE}(>1,000 \mathrm{IU} / \mathrm{ml})$ in $50 \%$ with a total $\mathrm{IgG}$ raised in $42 \%$ positivity for anti-A isohemaglutininas $(72.2 \%)$ and anti-B $(69.4 \%)$ in children.

The main cardiac disorders in toxocariasis are myocarditis, pericarditis, eosinophilic pericarditis, pericardial effusion and cardiac pseudotumors, and their frequency is unknown, with most cases described in adults. These complications occur due to larval invasion or secondary to inflammatory reaction and subsequent vascular obstruction leading to ischemia. Experimental studies show these changes after a week of infection. After 2 weeks of infection, granulomas can be observed with microabscesses present, myocytes with eosinophilia in the muscle fibers, and rapidly progressing to fibrosis and severe endomyocardial fibrosis. Various clinical manifestations such as syncope, dyspnea, chest pain, palpitations, fatigue, and pulsus paradoxus may suggest their presence $[8 \bullet \bullet]$.

The larval eye form has a low load, leading to low antibody production, and thus more free migration takes larvae affecting the eyes. Sanchez et al. [25] showed a prevalence of $0.57 \%$ ( $1: 512$ ELISA) of 175 children, average age of 6 years, most asymptomatic. Melewska-Mazur et al. [3] reported $3.6 \%$ of OLM [3] and Wiśniewska Ligier et al. [18••] $4.6 \%$ among 103 children who had retinitis, fibrotic lesions in the vitreous body, parafoveal lesion, unilateral or bilateral, and in less than $50 \%$ of cases had visual manifestations. The presence of loss of vision, strabismus and leukocoria suggests intraocular involvement [25]. Other authors in the evaluation of 11 children with OLM, between 2 and 17 years old, found posterior pole granuloma to be the most frequent, followed by peripheral and chronic endophthalmitis and had impairment of

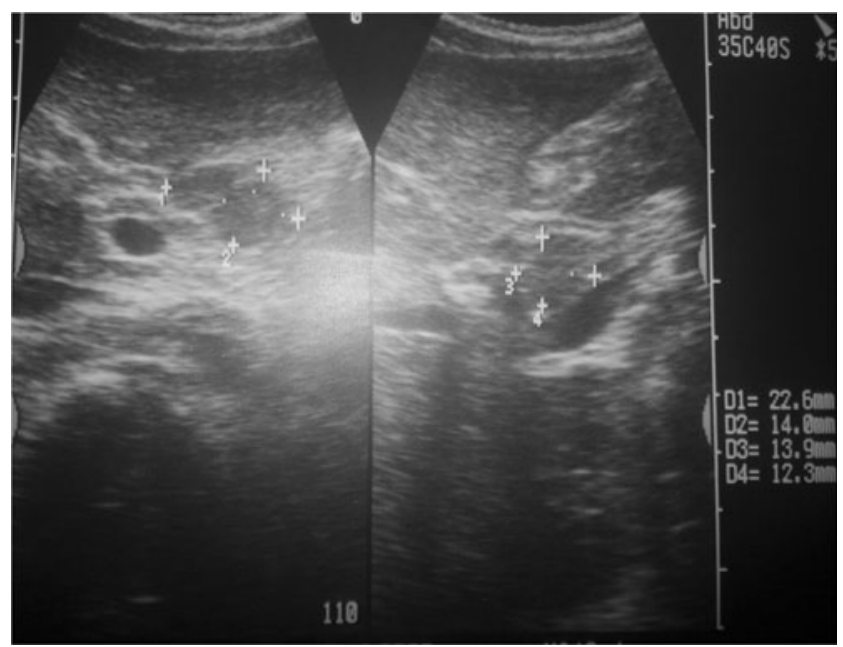

Fig. 1 Abdominal ultrasound showing hypoechoic liver lesions caused in a child with Toxocara canis infection visual acuity in $100 \%$ of them. Ocular ultrasound may aid in diagnosis in cases of opacity of the media, and in these cases showed retinal mass or granuloma, high reflectivity, which can be located in the posterior pole and periphery, calcified or not, having as main characteristic the presence of membranes vitreously attached [26]. We found a prevalence of $6 \%$ of OLM (unpublished).

Toxocariasis has been associated with asthma [27], asthmalike symptoms and high levels of specific IgE against aeroallergens $[20,28]$, serum total IgE, eosinophil count $[20$, 28], increased skin sensitivity to aeroallergens, atopic asthma in children and reduced [28] lung function and inversely proportional positive serology with skin prick test reactivity (30.4\% had at least a reaction to an allergen) and $22.4 \%$ had recent wheeze [20]. Other authors showed in asthmatics presence of elevated total IgE and positive prick test $[17,29]$. Toxocara spp. stimulate the $\mathrm{Th}_{2}$ immune response, a type of immune response that is considered to be central to the development of atopy and allergy. Experimental infections in mice with $T$. canis have been associated with elevated inflammatory activity, intense eosinophil migration to the lungs and plasma levels of high pro-inflammatory cytokines such as IL-6 and IFN-gamma [20].

Neurological toxocariasis infection can involve the central nervous system, such as meningitis, meningoencephalitis and transverse myelitis, but appears to be rare [30 $0^{\bullet}$. There are few reports of eosinophilia in the cerebrospinal fluid (CSF) of $27 \%$ and $35 \%$ in peripheral blood, and computed tomography scans demonstrating subtle changes in the density of the cerebellar region [31].

Covert toxocariasis is not rare and is characterized by a syndrome that includes chronic weakness, abdominal pain, several allergic signs often conjunctival or skin, such as skin rash or itching, diffuse myalgias, angioneurotic edema, cough, and sleep disorders and behavioral changes [30•].

The asymptomatic toxocariasis is characterized by positive serology, but the absence of eosinophilia and clinical symptoms $[2 \cdot \bullet]$.

\section{Diagnosis}

The diagnosis is based on clinical presentation, laboratory findings associated with serological that can be performed by the method of enzyme-linked immunosorbent assay (ELISA) or Western Blotting (WB) due to the difficulty of larvae in tissue biopsy $[2 \bullet \cdot 5 \bullet]$, besides being an invasive

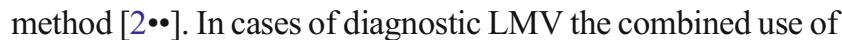
excretory-secretory antigens (TES)-ELISA has been confirmed by WB option with respect to sensitivity and specificity [30•]. Serology using TES is the best option for the laboratory diagnosis is considered a predictor of Toxocara canis infection when added to the relevant clinical data. 
Dosage of IgM by immunodiagnostic methods cannot be used for toxocariasis since IgM is found throughout the course of helminthiasis. Other immunoglobulins such $\operatorname{IgG} 4$ and $\operatorname{IgA}$ are unable to distinguish between active phase and selfhealing widespread infection [30•]. High concentration of $\operatorname{IgE}$ is the most active indicative toxocariasis [3]. Titers higher than 640 ELISA IgG anti-T.canis are related to possible cases of human toxocariasis, suggesting recent infection [2・•].

Currently, the diagnosis of covert toxocariasis relies on nonspecific symptoms associated with eosinophilia and/or high levels of eosinophil cationic protein (ECP) that were used to assess the presence of a current active toxocariasis in patients who did not display any blood eosinophilia [30•].

\section{Treatment and Clinical Monitoring}

Therapy should be instituted for all infected persons, even asymptomatic, due to possible complications in vital organs such as liver and eyes due to larval migration. The most commonly used drug is albendazole $(10 \mathrm{mg} / \mathrm{kg} /$ day, maximum $400 \mathrm{mg}$ ) once a day for 5-14 days) [2••]. Treatment is carried out with the OLM albendazole, which prevents the intraocular invasion of the parasite [25] for 14 days associated with oral corticosteroids and managed preferentially by an ophtalmologist.

In general, the evaluation of treatment efficacy for toxocariasis is quite difficult to assess due to the diverse clinical presentations. Few studies have been conducted to evaluate the effectiveness of antiparasitic drugs. Fernando et al. [32••] showed reduced levels of IgE and eosinophilia after the use of albendazole (administered at a dose of $50 \mathrm{mg} / \mathrm{kg} /$ day divided in two doses for 3 days) and diethylcarbamazine (6 mg/kg/day for 21 days) with significant decrease in the serological evaluation by ELISA titers in the albendazole group, but no difference was found among children between 4 and 13 years. Wiśniewska Ligier et al. [18••] studied treatment among three different groups that received two to three courses of anthelmintics (albendazole, $400 \mathrm{mg} /$ day) 3-5 days after 3 weeks, mebendazole (100-200 mg/day) for 3 days or 6 days. Antibody levels and eosinophils decreased after 4-6 months of treatment, significantly, however there was no correlation between the level of antibodies and eosinophils and time of assessment. However, high levels of IgG anti-Toxocara 45 months after treatment suggest ineffectiveness of treatment. $\operatorname{IgG}$ and $\operatorname{IgE}$ showed a decline after 4 years, and after 2 years the decline of $\operatorname{IgA}$ and maintenance of these markers after treatment may be due to reinfection, or reactivation of parasitic load [16••]. Another study of immigrants from Latin America showed a reduction of Toxocara spp. titers after 6 months of treatment [33].

An experimental study showed the contribution of probiotics in reducing the intensity of infection [34].
However, further studies are needed to evaluate proposed adjunct treatments for disease. Abdominal ultrasound and ophthalmologic evaluation should be performed after the diagnosis. If there is a change, liver ultrasound examination should be repeated monthly until complete resolution of lesions. The ophthalmic examination must follow the patient annually since the onset of the injury can be later.

The most important reason to provide antiparasitic and anti-inflammatory therapy is to achieve improvement of clinical symptoms. After the initial course of treatment, it is recommended to have sequential follow-up evaluations at 4 weeks and then after 3-6 months after resolution of symptoms and improvement of laboratory abnormalities. Laboratory monitoring is recommended with measurement of serum eosinophil measurement, quantitative levels of $\operatorname{IgE}$, and titers of $\operatorname{IgG}$ anti-Toxocara canis until normalization. If reinfection (either by maintaining contact with infected dogs concomitantly with rising eosinophilia or by the onset of new clinical symptoms) is suspected, a repeated course of antiparasitic therapy is recommended.

\section{Prevention}

In either urban, periurban, or rural settings where stray dogs are commonplace, educational and preventive interventions to reduce their numbers, such as through neutering procedures, may decrease opportunities for animal excreta from reaching public places where children or other individuals are exposed to Toxocara infection. Other important interventions include: having schools to close sand boxes; increase the use of fences to limit the entry of dogs and cats at local recreational areas [13]; prohibit animals on beaches and recreational places, or at least enforcing individuals in urban and periurban areas to strictly collect feces from their pets. In addition, there must be sanitary and legal control on companies that store and sell sand to places such as schools and playgrounds. Public authorities and families can work together to establish and perform safe and specific interventions and thus help prevent infection of children with Toxocara sp. The aim should also be to educate children with campaigns about personal hygiene when handling their pets or when playing at high-risk recreational sites. From a veterinary standpoint, the use of antiparasitic treatment for dogs at 2,4, 6 and 8 weeks old and at 6 months has shown to be an important public health intervention in highly endemic areas [35].

\section{Conclusions}

Toxocariasis is a parasitic infection with universal distribution but its prevalence is likely underestimated due to a lack of awareness of the impact of this parasitic infection in the 
community and by its recognition by clinicians. There are only a limited number of controlled clinical studies to address the impact of toxocariasis due to multiple factors, including the fact that most cases are not diagnosed, serologic assays have limitations, and there is an overall insufficient recognition of toxocariasis as an important parasitic infection from a public health perspective in many settings. New methodologies are being developed for the diagnosis of acute and chronic stages of the disease. Nevertheless, there is enough currently available evidence to support that early treatment helps reduce complications and morbidity.

\section{Compliance with Ethics Guidelines}

Conflict of Interest Elaine Alvarenga de Almeida Carvalho and Regina Lunardi Rocha declare that they have no conflict of interest

\section{References}

Papers of particular interest, published recently, have been highlighted as:

- Of importance

- Of major importance

1.• Núñez CR, Martínez GDM, Arteaga SY, et al. Prevalence and risk factors associated with Toxocara canis infection in children. Sci World J. 2013;2013:1-4. Prevalence of 22.2\% among 108 children 2-16 years, male, eosinophilia $>500 \mathrm{cells} / \mu \mathrm{l}$ and presence of dogs younger than 1 year in the household were statistically associated with positive serology $(O D>0.3)$.

2.• Carvalho EAA, Rocha RL. Toxocariasis: larvae visceral migrans in children. Pediatr (Rio J). 2011;87(2):100-10. Epidemiology, pathophysiology and clinical presentation in clinical syndromes in toxocariasis.

3. Avila LFC, Fonseca JSV, Dutra GF, et al. Evaluation of the immunosuppressive effect of cyclophosphamide and dexamethasone in mice with visceral toxocariasis. Parasitol Res. 2012;110:443-7. Immunosuppression stimulates increased larval burden in mice with toxocariasis.

4. Manini MP, Marchioro AA, Colli CM, et al. Association between contamination of public squares and seropositivity for Toxocara spp. in children. Vet Parasitol. 2012;188:48-52. Prevalence of $17.8 \%$ children between 1 and 12 years old in Paraná. The frequency of six to seven times per week for children in parks associated with seroprevalence. The majority presented eosinophilia geophagy and significantly associated.

5. Peixoto PL, Nascimento E, Cançado GGL, et al. Identification of candidate antigens from adult stages of Toxocara canis for the serodiagnosis of human toxocariasis. Mem Inst Oswaldo Cruz, Rio de Janeiro. 2011;106(2):200-6. The 58 and 68-kDa antigens might be most suitable for the immunodiagnosis of human toxocariasis.

6. Bolívar-Mejía A, Rodríguez-Morales AJ, Paniz-Mondolfi AE, et al. Cardiovascular manifestations of human toxocariasis. Arch Cardiol Mex. 2013;83:120-9.

7. Mattia S, Colli CM, Adami CM, et al. Seroprevalence of Toxocara infection in children and environmental contamination of urban areas in Paraná State, Brazil. J Helminthol. 2012;86:440-5. Prevalence of $36.8 \%, 353$ children aged zero to 12 years old $(0-5$ years) and persistent wheezing associated to serology; sex no association.

8.• Fragoso RP, Monteiro MBM, Lemos EM, et al. Anti-Toxocara antibodies detected in children attending elementary school in Vitoria, State of Espirito Santo, Brazil: prevalence and associated factors. Rev Soc Bras Med Trop. 2011;44(4):461-6. Prevalence of $51.6 \%$ among 391 children aged greater than or equal to 7 years, there was no statistical difference between sex and eosinophilia. ELISA (optical density $\geq 0.500$ ).

9.• Santarém VA, Chagas FN, Rubinsky-Elefant G, et al. Protective and risk factors for toxocariasis in children from two different social classes of Brazil. Rev Inst Med Trop Sao Paulo. 2011;53(2):67-72. Prevalence of $11.1 \%$ among 252 children aged 10 months to 15 years of age and that the presence of dogs was a risk factor for infection in the middle class and male sex was associated with variable disease.

10. Moura JVL, Santos SV, Castro JM, et al. Experimental study on vertical transmission of Toxocara cati larvae in Mus musculus. Arq Med Hosp Fac Cienc Med Santa Casa São Paulo. 2011;56(3):138-40.

11. Santarém VA, Pereira VC, Alegre BCP. Contamination of public parks in Presidente Prudente (São Paulo, Brazil) by Toxocara spp. eggs. Rev Bras Parasitol Vet, Jaboticabal. 2012;21(3):323-5. Of the parks, $96 \%$ were contaminated in the fall (statistically significant), but no association was found between temperature variation and number of eggs.

12. Khazan H, Khazaei M, Seyyed Tabace SJ, Mehrabi A. Prevalence of Toxocara spp. eggs in public parks in Tehran city, Iran. Iran J Parasitol. 72012(3):38-42. Contamination by Toxocara in 10\% of 120 parks. Of the parasites found, $51.9 \%$ were Toxocara spp.

13. Souza RF, Dattoli VCC, Mendonça LR, et al. Prevalence and risk factors of human infection by Toxocara canis in Salvador, State of Bahia, Brazil. Rev Soc Bras Med Trop. 2011;44(4):516-9.

14. Romero C, Mendoza G, Bustamante P, et al. Presencia y viabilidade de Toxocara spp em suelos de parques públicos, jardines de casas y heces de perros en Nezahualcóyotl, México. Rev Científica. 2011;21(3):1195-201. Toxocara canis present in regions of Mexico between 19 and $39 \%$, and around $69 \%$ of eggs are viable.

15. Marchioro AA, Colli CM, Ferreira EC, et al. Identification of public areas with potential toxocariasis transmission risk using geographical information systems. Acta Parasitol. 2013;58(3):328-33. Over 40\% contamination in areas on the periphery of cities, lawns and sand.

16.• Rubinsky-Elefant G, Hoshino-Shimizu S, Abe Jacob CM, et al. Potential immunological markers for diagnosis and therapeutic assessment of toxocariasis. Rev Inst Med Trop Sao Paulo. 2011;53(2):61-5. Twenty-seven children between zero and 12 years followed-up for 22 and 116 months. $\operatorname{IgG}$ and total $\operatorname{IgE}$ remained present for 4 years and IgA present for 2 years. $\operatorname{IgG}>205 \mathrm{kDa}, \operatorname{IgA}$ and IgE 29-38, 48-54, 81-93 kDa 95-121 kDa may be candidates for therapeutic markers.

17. Guilherme EV, Marchioro AA, Araujo SM, et al. Toxocariasis in children attending a public health service pneumology unit in Paraná State, Brazil. Rev Inst Med Trop Sao Paulo. 2013;55(3): 189-92. Prevalence of $4.2 \%$ (7) among 167 children aged zero to 15 years; two cases of moderate eosinophilia and five with positive reactions in allergy testing.

18.• Wiśniewska-Ligier M, Woźniakowska-Gęsicka T, SobolewskaDryjańska, et al. Analysis of the course and treatment of toxocariasis in children - a long-term observation. Parasitol Res. 2012;110:2363-71. Rate of 4.6\% among 103 children.

19. Sariego I, Kanobana K, Junco R, et al. Frequency of antibodies to Toxocara in Cuban schoolchildren. Trop Med Int Health. 2012;17(6):711-4. Study 1011 Cuban children from 5 to 11 years showed a prevalence of $38.8 \%$ and were more frequent between 7 and 10 years (diagnosed by ELISA).

20. Mendonça LR, Veiga RV, Dattoli VCC, et al. Toxocara seropositivity, atopy and wheezing in children living in poor neighborhoods 
in urban Latin American. PLoS Negl Trop Dis. 2012;6(11):e1886. Prevalence of $47 \%$ among 1445 children, 4 and 11 years, seropositivity was associated with eosinophilia $>4 \%$ and $>10 \%$ and total $\operatorname{IgE} \geq 0.35 \mathrm{kU} / 1$ and specific IgE to aeroallergens.

21. Negri EC, Santarém VA, Rubinsky-Elefant G, et al. Anti-Toxocara spp. antibodies in an adult healthy population: serosurvey and risk factors in Southeast Brazil. Asian Pacific J Trop Biomed. 2013;3(3): 211-6. Prevalence of $8.7 \%$ of 19- to 65-year-olds, contact with soil was a risk factor for infection and age was not statistically significant.

22. Dattoli VCC, Freire SM, Mendonça LR, et al. Toxocara canis infection is associated with eosinophilia and total $\mathrm{IgE}$ in blood donors from a large Brazilian centre. Trop Med Int Health. 2011;16(4):514-7. Children aged 4-11 years were studied, of whom $47 \%$ were seropositive for anti-Toxocara IgG; eosinophilia $>4 \%$ occurred in $74.2 \%$ and $>10 \%$ in $25.4 \% ; 59.6 \%$ had elevated levels of total IgE; anti-Toxocara IgG was positively associated with elevated eosinophils counts, total IgE, and the presence of specific $\operatorname{IgE}$ to aeroallergens, but was inversely associated with skin prick test reactivity.

23. Maraghi S, Rafiei A, Hajihossein R, et al. Seroprevalence of toxocariasis in hypereosinophilic individuals in Ahwaz, southwestern Iran. J Helminthol. 2012;86:241-4. Prevalence (Western blot) of $19 \%$ in adults $>20$ years and associated with eosinophilia $>10 \%$.

24. Mazur-Melewska K, Mania A, Figlerowicz M, et al. The influence of age on a clinical presentation of Toxocara spp. infection in children. Ann Agric Environ Med. 2012;19(N 2):233-6. Higher prevalence (ELISA IgG $>11 \mathrm{UI} / \mathrm{ml}$ ) among children aged $7-10$ years from 84 children. In children below 3 years, eosinophilia was more frequent (average 4,023 cells/ $\mathrm{mm}^{3}$ ), elevated IgE levels (average of $659)$ and $\operatorname{IgG}$ (mean 1,15I), but there was no statistical significance in relation to serology. Moreover, we found no correlation between age and clinical symptoms. The most frequent findings were lymphadenopathy (37\%), hepatomegaly in 18\%, retinal granulomatous $3.6 \%$.

25. Sánchez TJE, López GJP, González NM, et al. Detección de lesioes oculares en niños seropositivos para Toxocara canis. Rev Chil Infect. 2011;28(5):431-4. One hundred and seventy-five children with a mean age of 6 years were infected (ELISA ranged from 1:64 A 1:1,024); gender was not statistically associated with toxocariasis; a child of 7 years $(0.57 \%)$ had ocular injury with loss of vision, 1:512 ELISA.
26. Morais FB, Maciel AL, Farias TE, et al. Ultrasonographic findings in ocular toxocariasis. Arq Bras Oftalmol. 2012;75(1):43-7. Contribution of ultrasonography in the diagnosis of ocular toxocariasis, when no media opacity to ophthalmologic examination was found in 11 patients.

27. Kanobana K, Vereecken K, Diaz RJ, et al. Toxocara seropositivity, atopy and asthma: a study in Cuban schoolchildren. Trop Med Int Health. 2013;18(4):403-6. A study of 958 school children found $40.1 \%$ Toxocara-seropositive. Prevalences were $21.7 \%$ for current wheeze and $32.7 \%$ for physician-diagnosed asthma.

28. Walsh MG. Toxocara infection and diminished lung function in a nationally representative sample from the United States population. Int J Parasitol. 2011;41:243-7.

29. Machioro AA, Colli CM, Mattia S, et al. Eosinophilic count and soropositivity for IgG antibodies to Toxocara spp. in children assisted at the public health service. Rev Paul Pediatr. 2011;29:80-4.

30. Fillaux J, Magnaval J-F. Laboratory diagnosis of human toxocariasis. Vet Parasitol. 2013;193:327-36. Immunodiagnostic and difficulties to define recent illness.

31. Salvador S, Ribeiro R, Winckler MI, et al. Pediatric neurotoxocariasis with concomitant cerebral, cerebellar, and peripheral nervous system involvement: case report and review of the literature. J Pediatr (Rio J). 2010;86:531-4.

32.• Fernando SD, Wickramasinghe VP, Dewasurendra RL, et al. Comparative effect of albendazole and diethylcarbamazine in the treatment of toxocariasis in children from Sri Lanka: a preliminary study. J Clin Med Res. 2011;3(3):46-51. The reduction of serum levels eosinophilia was statistically significant in children treated with albendazole. There was no statistically significant difference between groups.

33. Turrientes M-C, Ayala CAP, Norman F, et al. Visceral larva migrans in immigrants from Latin America. Emerg Infect Dis. 2011;17(7): 1263-5.

34. Avila LFC, Conceição FR, Telmo PL, Dutra GF, Santos DG, Martins LHR, et al. Saccharomyces boulardii reduces infection intensity of mice with toxocariasis. Vet Parasitol. 2012;187:33740. Probiotic reduces the intensity of infection by $41.9 \%$ in mice with toxocariasis.

35. Deplazes P, van Knapen F, Schweiger A, et al. Role of pet dogs and cats in the transmission of helminthic zoonoses in Europe, with a focus on echinococcosis and toxocarosis. Vet Parasitol. 2011;182: $41-53$. 